ReLATO DE CASO

CASE REPORT

\title{
Esclerose Sistêmica e Sarcoidose ${ }^{(*)}$
}

\section{Scleroderma and Sarcoidosis}

\author{
Fernanda Guidolin ${ }^{(1)}$, Letícia Esmanhotto ${ }^{(2)}$, Marilia B. Silva ${ }^{(3)}$, Lismari Mesquita ${ }^{(4)}$, Thelma L. Skare ${ }^{(5)}$
}

\section{RESUMO}

Os autores descrevem o caso de uma paciente com esclerose sistêmica (ES) - forma limitada - com comprometimento pulmonar tipo fibrose intersticial. Após sete anos sem acompanhamento, foram identificados gânglios mediastinais e esplenomegalia. A biópsia de linfonodos mostrou granuloma não caseoso sugestivo de sarcoidose. Estamos mostrando, neste caso, a associação de ES e sarcoidose, para chamar a atenção para esse fato e enfatizar que a sarcoidose deve ser lembrada no diagnóstico diferencial das complicações pulmonares da esclerodermia.

Palavras-chave: esclerose sistêmica, esclerose sistêmica limitada, fibrose intersticial pulmonar, sarcoidose.

\section{INTRODUÇÃO}

A esclerodermia (ES) é caracterizada clinicamente por fenômeno de Raynaud (vasoespasmo reativo diante de estresse, principalmente frio) e espessamento de pele, podendo acometer órgãos internos tais como esôfago e pulmão. $\mathrm{O}$ envolvimento pulmonar é uma das formas comuns de lesão visceral, só sendo superada, em freqüência, pelas lesões esofágicas $^{(1)}$.

O envolvimento pulmonar da ES é bastante diversificado, podendo aparecer como infiltrados intersticiais, hipertensão pulmonar, micronódulos, fibroses pleurais e pneumonias $\operatorname{aspirativas}^{(1,2)}$.

A fibrose intersticial é a forma mais comum de envolvimento pulmonar e sua prevalência varia entre 25\% e 90\% - variabilidade esta que está na dependência do perfil étnico

\begin{abstract}
The authors describe the case of a patient with limited scleroderma and interstitial lung disease. Follow-up was lost for seven years, when patient returned presenting nodular mediastinal enlargement and splenomegaly. Lymph node biopsy showed granulomatous lesions without caseum suggestive of sarcoidosis.

This case is being presented to remind the association of scleroderma and sarcoidosis as a possible differential diagnosis of scleroderma pulmonary complications.
\end{abstract}

Keywords: scleroderma, interstitial pulmonary fibrosis, sarcoidosis.

da população estudada, assim como dos métodos utilizados para a sua detecção ${ }^{(3)}$. Os auto-anticorpos apresentados pelo paciente guardam associação com seu aparecimento, sendo esta complicação mais comum nos portadores de anticorpo anti Scl-70 (ou anti DNA topoisomerase-1) e mais rara nos portadores de anticorpos anticentrômero ${ }^{(3)}$. Sua prevalência é também ligeiramente mais comum na forma difusa da doença quando comparada com a forma limitada. Um estudo feito por Steen mostra envolvimento de $40 \%$ dos pacientes com forma difusa contra $23 \%$ na forma limitada quando se utiliza disfunção ventilatória restritiva como elemento marcador de seu aparecimento ${ }^{(4)}$.

O envolvimento pulmonar esclerodérmico tende a surgir, em geral, dentro dos três primeiros anos do início da doença ${ }^{(3)}$ e o seu achado aumenta a morbimortalidade destes pacientes. Por esta razão, postula-se que portadores

\footnotetext{
* Trabalho realizado no Serviço de Reumatologia do Hospital Universitário Evangélico de Curitiba (HUEC), Curitiba, PR, Brasil. Recebido em 22/03/2004. Aprovado, após revisão, em 10/01/2005.

1. Residente (R-2) de Reumatologia do HUEC.

2. Residente (R-1) de Reumatologia do HUEC.

3. Professora assistente de Reumatologia do Curso de Medicina da Faculdade Evangélica do Paraná.

4. Professora assistente de Patologia Clinica do Curso de Medicina da Faculdade Evangélica do Paraná

5. Professora assistente de Reumatologia do Curso de Medicina da Faculdade Evangélica do Paraná.
}

Endereço para correspondência: Thelma L Skare. Rua João Alencar Guimarães,796, CEP 80310-420. Curitiba, PR, Brasil. E-mail: tskare@onda.com.br 
de esclerodermia devam ser acompanhados anualmente com tomografia de alta resolução e/ou testes de função respiratória nos primeiros anos de doença ${ }^{(3)}$.

Todavia, o achado de um envolvimento pulmonar intersticial na esclerodermia não é necessariamente secundário a esta doença, como ilustra o caso descrito. A sarcoidose pode se manifestar em pacientes com esclerodermia $(\mathrm{SA})^{(1,5-8)}$, devendo ser considerada no diagnóstico diferencial de processo intersticial pulmonar desta afecção e conseqüente direcionamento terapêutico.

\section{RELATO DE CASO}

Trata-se de paciente do sexo feminino, parda, 57 anos de idade, em acompanhamento pelo Ambulatório de Reumatologia do Hospital Universitário Evangélico de Curitiba por esclerodermia. Sua esclerodermia havia sido diagnosticada há cinco anos, quando a paciente apresentou queixas de alterações vasculares compatíveis com fenômeno de Raynaud, fraqueza muscular (principalmente em cinturas escapular e pélvica), disfagia a alimentos sólidos e artralgias migratórias. Apresentava hipotireoidismo controlado com $50 \mu \mathrm{g}$ de tiroxina/dia. Trabalhava na lavoura e não era fumante. $\mathrm{O}$ exame físico feito naquela época revelou esclerose da face e dos membros até cotovelos e joelhos; afilamento de polpas digitais com cianose. Capilaroscopia mostrou ectasia e desvascularização focal e difusa (padrão $\mathrm{SD})$. O Fator antinúcleo (FAN) foi positivo no padrão nucleolar (1/320); pesquisas de anticentrômero e anti Scl70 resultaram negativas. Dosagem de creatinoquinase $(\mathrm{CK})=$ $473 \mathrm{U} / 1(\mathrm{nl}=165 \mathrm{U} / \mathrm{l})$ e transaminase glutâmico oxalacética (TGO) de $92 \mathrm{UK} / 1$ (nl = 36UK/L). Hemograma mostrava uma anemia normocrômica e normocítica com volume

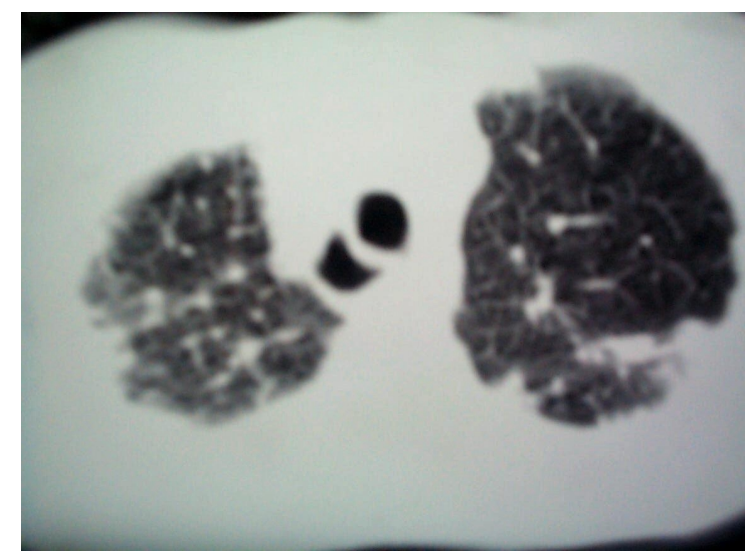

FiguRa 1 - Tomografia computadorizada de tórax mostrando nódulos predominantemente em lobos superiores globular de 35\%. Velocidade de hemossedimentação (VHS) $=47 \mathrm{~mm}$ na $1^{\mathrm{a}}$ hora. $\mathrm{O}$ esofagograma era normal assim como radiografia do tórax, espirograma e ecocardiograma. A paciente foi tratada com nifedipina e metoclorpramida e com corticóides inicialmente em doses altas (pelo quadro de miosite) e, depois, em doses sucessivamente menores, com melhora da força muscular e dos sintomas vasculares periféricos.

Por dificuldades socioeconômicas a paciente não compareceu aos controles estipulados, só retornando 2,5 anos mais tarde. Durante este período havia feito uso de nifedipina e tiroxina de maneira irregular. Queixava-se de disfagia a sólidos, dor epigástrica em queimação, falta de ar aos grandes e médios esforços e tosse seca. Ao exame físico persistia com esclerose de pele até cotovelos e joelhos além da face. Apresentava estertores crepitantes em ambas as bases dos pulmões. No abdome palpava-se uma ponta do baço. Exames mostraram ecocardiograma normal e esôfago alargado com retardo no esvaziamento e distúrbio na motilidade ao esofagograma. A endoscopia mostrava esofagite grau I. A tomografia computadorizada do tórax (TAC) mostrava presença de nódulos irregulares subpleurais principalmente em lobos superiores (Figura 1), lesões intersticiais com faveolamento e áreas em "vidro despolido" em ambos os pulmões, sobretudo em bases (Figura 2) e conglomerados de linfonodos mediastinais, hilares com calcificações de permeio (Figura 3). O VHS era de $30 \mathrm{~mm}$ na $1^{\mathrm{a}}$ hora, hematócrito de 36\% e PPD negativo. Provas de função ventilatória mostraram distúrbio restritivo leve, reserva ventilatória diminuída e volume de reserva expiratória diminuída sem resposta a broncodilatador. Uma mediastinoscopia com biópsia de linfonodo foi realizada e o exame histológico mostrou granulomas não caseosos compatíveis

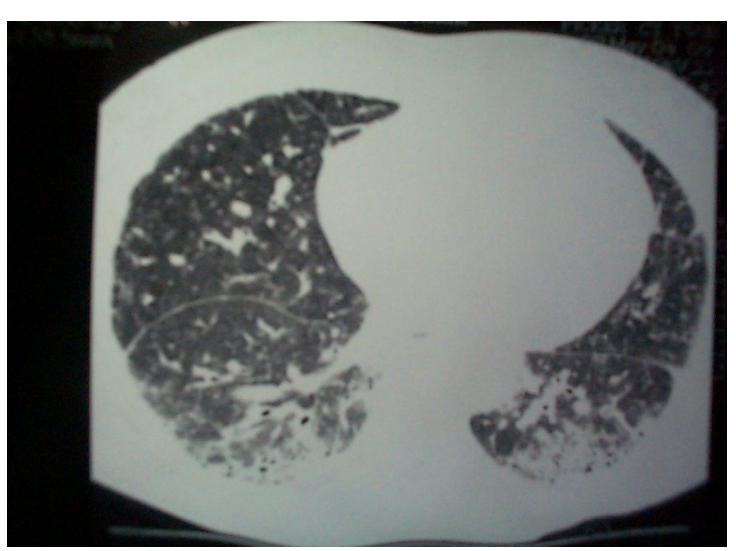

FIgURA 2 - Tomografia computadorizada de tórax mostrando faveolamento e áreas de "vidro despolido" predominando em lobos inferiores 


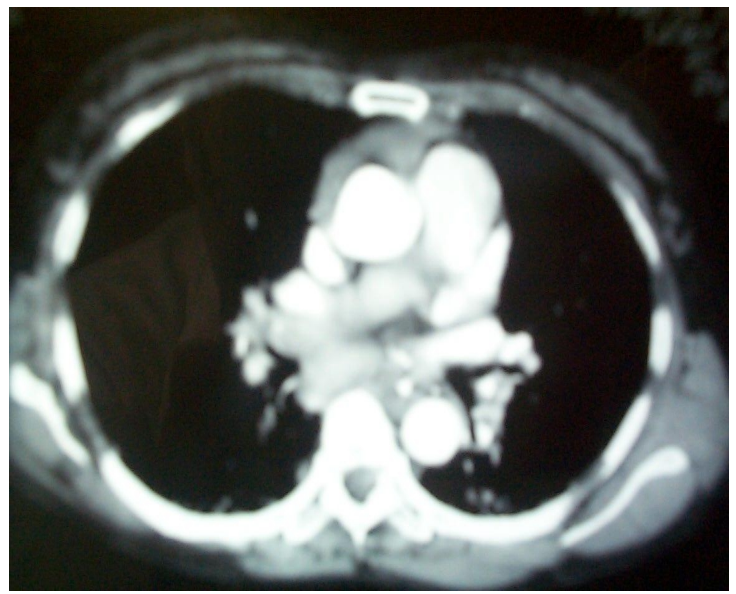

FIgURA 3 - Tomografia computadorizada de tórax mostrando conglomerados de linfonodos mediastinais e hilares

com o diagnóstico de sarcoidose (Figura 4). A paciente foi tratada com corticóide e ciclofosfamida em pulsos. Após seis meses de tratamento, ela sente-se subjetivamente melhor. TAC de tórax e as provas de função ventilatória permaneceram inalteradas.

\section{DISCUSSÃO}

A associação entre sardoidose (SA) e esclerose sistêmica (ES) tem sido notada por diversos autores, embora não se tenha encontrado, até o momento, uma explicação plausível para a mesma ${ }^{(5-8)}$. Os casos descritos na literatura denotam existir tanto um aparecimento simultâneo das duas doenças como o fato de qualquer uma delas poder ser o advento inicial $^{(6,7)}$. A associação tem sido encontrada tanto com a forma limitada como com a forma difusa da esclerose sistêmica ${ }^{(6,7)}$.

Vários antígenos de histocompatibilidade (HLA) têm sido apontados como predisponentes tanto à esclerose sistêmica como à sarcoidose e, dentre eles, o HLA DR3 é notado nas duas doenças ${ }^{(3,9,10)}$, sendo um elo comum aos seus aparecimentos.

Como as duas enfermidades podem se apresentar com fibrose intersticial pulmonar e linfadenomegalias mediastinais, fica dificil estabelecer qual a verdadeira causa da lesão pulmonar naquele que porta as duas doenças. Entretanto, alguns dados podem ser utilizados ao se tentar esta diferenciação. Enquanto o processo intersticial na ES tende a ser subpleural e basal ${ }^{(1)}$, o da SA é predominante em lobo superior $^{(11)}$. As linfadenomegalias são mais comuns nesta última,
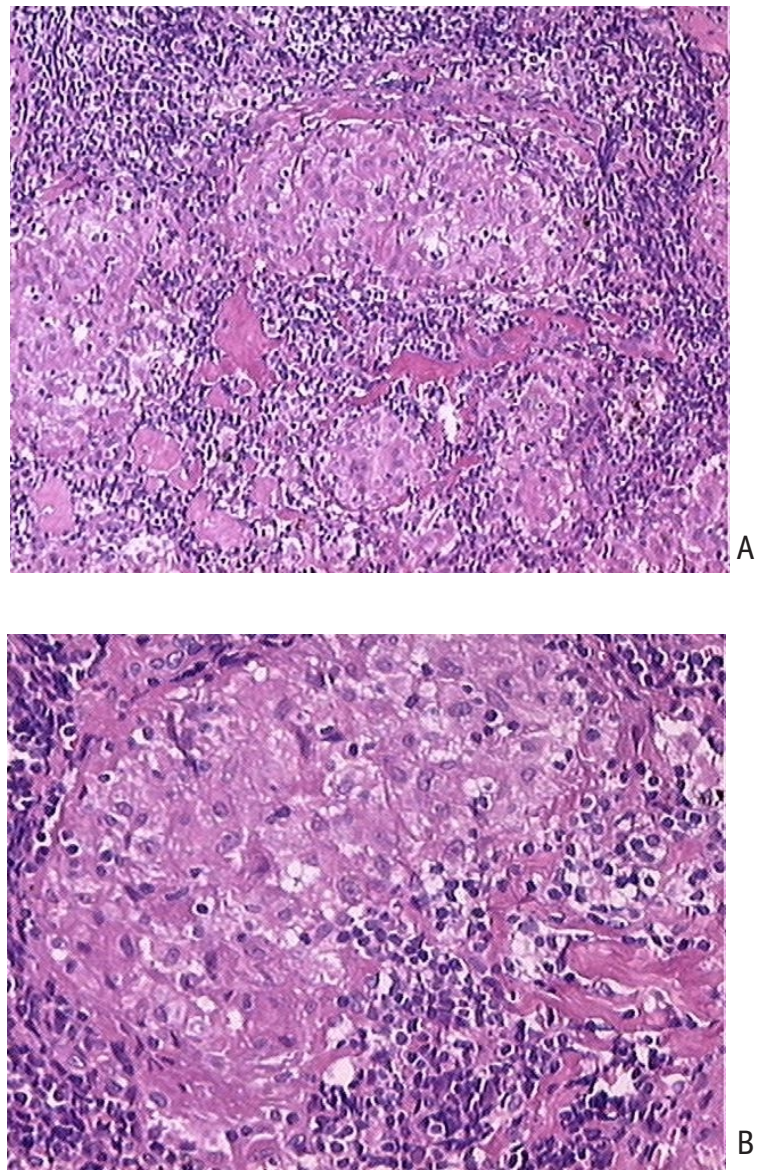

Figura 4 - A (aumento de 100X) e B (aumento de 400X). Estudo anatomopatológico de linfonodo mediastinal mostrando granulomas não caseosos compatíveis com sarcoidose

acontecendo em $75 \%$ a $90 \%{ }^{(12)}$ dos casos, enquanto na ES elas são esperadas em torno de $40 \%$ a $58 \% \%^{(3)}$. O lavado broncoalveolar, que por razões técnicas não pôde ser realizado nesta paciente, é também um elemento de diferenciação. $\mathrm{Na}$ SA, ele mostra um predomínio de linfócitos (com um aumento da razão entre células $\left.\mathrm{T} \mathrm{CD}^{+}: \mathrm{CD}^{+}\right)^{(11)}$; na ES existem principalmente neutrófilos e eosinófilos, sendo os linfócitos encontrados apenas na fase inicial do processo $^{(1)}$.

No caso descrito, a ausência do anticorpo anti Scl-70, a forma limitada da doença, o aparecimento da lesão pulmonar intersticial relativamente tardio, ou seja, após 7,5 anos depois do diagnóstico (embora este dado seja impreciso pela falta de acompanhamento adequado da paciente), o fato de o aparecimento da lesão pulmonar ocorrer junto com a esplenomegalia e o achado de granulomas nos linfonodos mediastinais favorecem o diagnóstico de SA 
como causa etiológica da doença intersticial, embora se tenha plena consciência de que tais dados são tênues e não permitem afirmação categórica.

Bandt et al., que descreveram esta associação em cinco pacientes, notaram que o uso de corticoterapia como forma isolada de tratamento mostrou pouca eficiência e que seus pacientes tiveram um prognóstico grave, com dois deles evoluindo para óbito ${ }^{(7)}$. Estes mesmos autores propunham o uso do metotrexato como uma forma alternativa de tratamento, uma vez que tal medicamento tem se mostrado eficiente nas duas doenças em questão. Entretanto, tais autores não levaram em consideração a possibilidade da

\section{REFERÊNCIAS}

1. Minai AO, Dweik RA: Manifestations of scleroderma pulmonary disease. Clin Chest Med 19: 713-28, 1998.

2. Franquet T: High resolution $\mathrm{CT}$ of lung disease related to collagen vascular disease. Radiol Clin North Am 39: 1171-87, 2001.

3. White B: Interstitial lung disease in scleroderma. Rheum Dis Clin North Am 29: 371-90, 2003.

4. Steel VD: Severe restrictive lung disease in systemic sclerosis. Arthritis Rheum 37: 1283-9, 1994.

5. Arapis J, Kaklamani V, Rapti A, Anagnostopoulou U: A case of scleroderma associated with sarcoidosis. Clin Exp Rheumatol 15: 337-8, 1997.

6. Cox D, Conant E, Earle L: Sarcoidosis in systemic sclerosis: report of 7 cases. J Rheumatol 22: 881-5,1995.

7. Bandt M, Perrot S, Masson C, Meyer O: Systemic sclerosis and sarcoidosis, a report of five cases. BrJ Rheumatol 36 :117-9, 1997.

8. Groen H, Postma DS, Kallenberg CGM: Interstitial lung disease and myositis in a patient with simultaneously occurring sarcoidosis and scleroderma. Chest 104: 1298-300, 1993. pneumonite por metotrexato, que, mesmo não sendo um efeito colateral comum, pode trazer mais dificuldades para acompanhar o curso de futuros eventos ${ }^{(13)}$. Outras drogas propostas para o tratamento da complicação pulmonar, tanto da sarcoidose como da esclerodermia, incluem ciclofosfamida e azatioprina ${ }^{(14,15)}$.

Neste caso optou-se pela ciclofosfamida associada ao corticóide, com estabilização do quadro.

A superposição de duas doenças relativamente raras aponta para a possibilidade de elementos etiológicos comuns, que ao serem investigados poderão vir a auxiliar o entendimento da patogênese das mesmas.

9. Fontenot A, King Jr TE: Pathogenesis of sarcoidosis. In Rose B. Uptodate.com, 2005. Capturado em http://www.uptodate.com.

10. Du Bois RM: The genetic predisposition to interstitial lung disease. Chest 121: 14S-20S, 2002.

11. King Jr TE: Overview of sarcoidosis. In Rose B. Uptodate.com, 2005. Capturado em http://www.uptodate.com.

12. Bein ME, Putman CE, Mc Loud, et al: A re-evaluation of intrathoracic lymphadenoparty in sarcoidosis. Am J Radiol 131: 409-15, 1978.

13. St Clair EW: Disease modifuing antirheumatic drugs. In Klippel JH, Crofford LJ, Stone JH, Weyand CM (Eds): Primer on the Rheumatic Diaseases; 2001. Arthritis Foundation, Atlanta, 599-605.

14. King Jr TE: Treatment of pulmonary sarcoidosis with alternative to corticosteroids. In Rose B. Uptodate.com, 2005. Capturado em http://www.uptodate.com.

15. Clements PJ: Systemic sclerosis: Treatment. In Klippel JH, Crofford LJ, Stone JH, Weyand CM (Eds): Primer on the Rheumatic Diaseases; 2001. Arthritis Foundation, Atlanta, 364-7. 